\title{
Bulk superconducting tube subjected to the stray magnetic field of a solenoid
}

\author{
K Hogan, J-F Fagnard, L Wéra, B Vanderheyden and P \\ Vanderbemden \\ University of Liège, SUPRATECS research group, Department of Electrical \\ Engineering \& Computer Science (B28), Sart-Tilman, B-4000 Liège, Belgium \\ E-mail: kevin.hogan@ulg.ac.be
}

\begin{abstract}
Hard type-II hollow superconductors are well suited for low frequency magnetic shielding. The properties and performances of superconducting magnetic shields subjected to homogeneous magnetic fields have been extensively discussed in the literature. In the present work, we investigate the magnetic shielding and the penetration of magnetic flux in a bulk high temperature superconducting tube subjected to the inhomogeneous fringe field of a solenoidal coil. Thanks to a bespoke microdisplacement measurement system, we measure the magnetic field distribution around the tube. We develop a full 3D finite element model based on an $\mathrm{H}$ formulation to understand the flux penetration mechanisms and predict the shape of the current loops. Using constitutive law parameters obtained from previous independent experiments, our model is found to be in excellent agreement with the measurements. We discuss how to assess the degree of inhomogeneity of the magnetic field and show that, in our case study, the field can be treated as the magnetic field of an equivalent magnetic dipole. We also show that some features of the flux penetration in inhomogeneous field can be also observed when the tube is subjected to an oblique homogeneous magnetic field, which offers a better understanding of the shielding current density distribution inside the shield. Finally, we discuss the magnetic field concentration occurring around the shield for different magnetic field configurations. In particular, we show that the extremities of the tube on the side not facing the magnetic field source experience the highest flux concentration.

DOI: https://doi.org/10.1088/1361-6668/aa9651
\end{abstract}

Published in Supercond. Sci. Technol. on 21 November 2017 


\section{Introduction}

The penetration of magnetic flux lines in hard type-II superconductors subjected to a uniform magnetic field can be understood in the framework of the Bean model [1] for a variety of geometries [2, 3, 4, 5]. Under an increasing external field, the general picture is that the strong pinning of flux lines gives rise to shielding currents flowing at the periphery of the superconductor and leaving a flux free region in the central part. If the superconductor has a hollow geometry, the central part can be accessed by the user and corresponds to a zone where the magnetic field is strongly attenuated, hence constituting a very efficient magnetic shield [6]. Superconducting shields offer a number of advantages over conventional ferromagnetic shields, in particular (i) the shielding of high magnetic field amplitudes [7, 8], (ii) the possibility of creating lightweight structures, (iii) large magnetic field attenuations [9] and (iv) the possibility to couple them with ferromagnetic materials to create so called cloaking devices [10] i.e. objects which shield a given magnetic field with no perturbation of the nearby magnetic field.

Hollow superconducting materials used as magnetic shields have been studied mainly under a homogeneous applied magnetic field or in situations where the shielding current path is a priori known. The various shapes investigated include tubes [8, 11, 12, 13, 14], plates [15], tapes [16, 17, 18], cups [19, 20], as well as structures made of different elements. In a homogeneous magnetic field the cause of the physical phenomenon, i.e. the magnetic field to shield, is known a priori everywhere and can be defined easily. Consequently, apprehending the distribution of the superconducting currents within the superconductor can be relatively straightforward since the flux lines approach the superconductor with a certain degree of symmetry.

In practical applications, however, such homogeneous magnetic fields are rarely encountered. A sensitive equipment, for instance a cryocooler [21] or a measurement instrument [14], requiring shielding from the magnetic field of a source localized in its vicinity experiences an inhomogeneous magnetic field, e.g. next to MRI machines or particle accelerators [22]. This corresponds to much more intricate situations since the source of magnetic field is now applied sideways. This often leads to a total loss of symmetry of the problem except in particular situations, e.g. a cylindrical coil placed coaxially to a cylindrical superconductor [23]. The consequence is that the general shape of the shielding current loops cannot be predicted intuitively. Moreover, possible local field concentrations deteriorating the shielding properties [23] cannot easily be located. Although superconductors subjected to inhomogeneous fields have been investigated extensively for understanding superconducting levitation and studying magnetic bearing applications, (i.e. situations involving a permanent magnet and a plain bulk superconductor, see e.g. Refs. [24, 25]), a study aiming at elucidating the magnetic shielding process of a hollow superconductor subjected to a nearby source is strongly required. In the present work, we investigate the principal features of the magnetic response of 


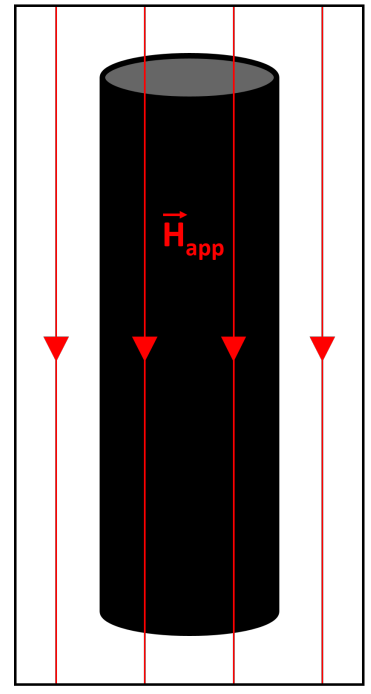

(a)

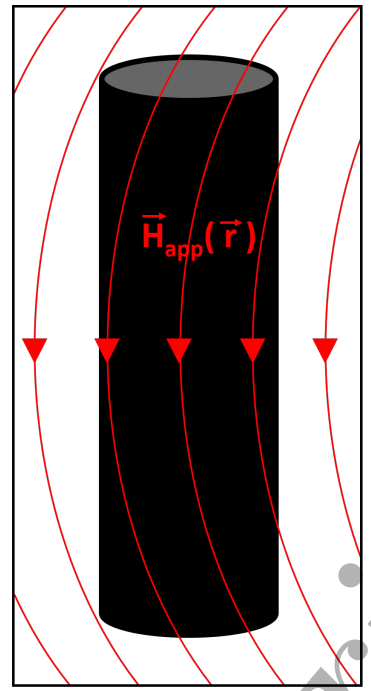

(b)

Figure 1. Schematic illustration of a superconducting tube subjected to (a) a homogeneous magnetic field and (b) the fringe field of a nearby magnetic source

a superconducting tube subjected to the inhomogeneous magnetic field generated by a nearby solenoid electromagnet. Figure 1 illustrates schematically the difference between (a) an axial homogeneous magnetic field and (b) the stray field of a magnetic field source placed in the vicinity of the hollowsuperconducting tube. The inhomogeneity of the magnetic field has two direct consequences: first, there is a variation of applied magnetic field strength i.e. the local magnetic flux density along the surface depends on the position; second, magnetic flux lines are curved and the shield is in general subjected to both axial and transverse components of magnetic field. These two features are linked intrinsically [26]: if $|\mathbf{B}|$ denotes the strength of flux density and $\mathbf{b}$ a unit vector along $\mathbf{B}$, the relation $\operatorname{div} \mathbf{B}=0$ leads to $(\operatorname{grad}|\mathbf{B}|) \cdot \mathbf{b}+|\mathbf{B}|(\operatorname{div} \mathbf{b})=0$, i.e. if the flux density decreases when one moves along a field line (i.e. the first term is negative), the local unit vectors should diverge (i.e. $\operatorname{div} \mathbf{b}>0$ ). In the simple situation illustrated in Figure 1 (a), the increasing applied field generates azimuthal currents leading to a circular flux front in the walls of the superconductor and local field enhancement near the open ends [13. In the configuration shown in Figure 1 (b), the two principal questions that will be addressed in this paper concern (i) the exact shape of the flux front and (ii) the locations where the local applied field is the strongest, i.e. the zones where the easiest entry gates are located.

In order to characterize the magnetic shielding effectiveness of superconducting magnetic shields, two parameters are generally used: the shielding factor, $S$, and the threshold induction, $B_{\text {lim }}$. The shielding factor is defined as the ratio of the applied magnetic flux density field, $B_{a p p}$, over the measured flux density inside the shield, $B_{\text {meas }}$. This parameter is, in general, position dependent since the field produced by the mag- 
netic shielding currents is non-uniform, irrespective of whether the applied field is uniform or not. Since the shielding factor decreases as the applied field increases, the threshold induction, $B_{\text {lim }}$, is usually defined as the maximum induction one can apply before the shielding factor $S$ falls below a given threshold value. In the context of a non-homogeneous applied field, the main question is how to define the applied field required to determine both $S$ and $B_{\text {lim }}$. In the present work, we define the reference applied magnetic field as the magnetic field that would be measured at the centre of the superconducting tube when it is in the normal state. Our choice is consistent with the one we have employed in a simpler situation where the magnetic field source is placed inside and centred within the superconducting tube [23]. This choice is also consistent with the fact that, when an uniform magnetic field is applied, the best shielding occurs at the centre of the tube and the device to be protected should be placed at this location.

This paper is structured as follows: In Section 2, we introduce the main parameters of the studied geometry and the experimental set-up. The modelling tools developed to obtain information on the current density distribution and the magnetic field inside the sample are detailed in Section 3 . Section 4 is dedicated to the experimental and numerical results. These results are discussed in Section 5 and conclusions are drawn in the last section.

\section{Geometry and experimental set-up}

\subsection{Studied geometry}

We consider a superconductor in the form of a hollow cylinder that is placed in the fringe field of a superconducting exciting coil. The exciting coil is a finite length solenoid. We consider first a reference geometry that is studied both experimentally and numerically in Section 4. Then the influence of the aspect ratio (length / diameter) of the coil will be investigated numerically in Section 5 .

Figure 2 details how the sample and the exciting coil are positioned in the reference geometry. The hollow superconducting cylinder is placed with its axis parallel to the main axis of the exciting coil. In order to simplify the analysis by taking advantage of the symmetry, we choose to have the centre of the tube in the median plane of the coil. The dimensions of the exciting coil are chosen to be representative of a situation that could be encountered in practice. If $a$ and $2 b$ denote respectively the average radius and the length of the exciting coil, its aspect ratio $(b / a)$ is 3.2 . In our reference geometry, the centre of the superconducting tube is placed at a distance $(8 a)$ from the axis of the solenoid. In so doing, we ensure that the maximum applied magnetic field can exceed the full-penetration field of the superconductor. The superconducting hollow cylinder has an aspect ratio of 3.5, i.e. close to that of the exciting coil, with the following dimensions: height, $h=80 \mathrm{~mm}$; wall thickness, $t=1.6 \mathrm{~mm}$ and inner diameter, $d=21$ 


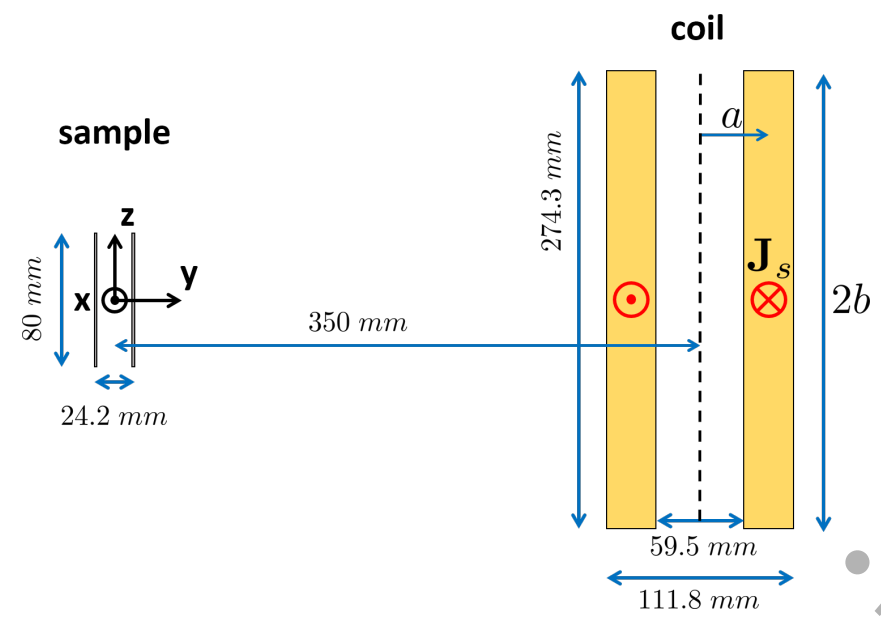

Figure 2. Disposition of the superconducting hollow cylinder and the exciting coil in the reference geometry that is studied both experimentally and numerically. The superconductor and the cylinder are annotated with their dimensions. The main axes of both cylinders are in the $y z$-plane and parallel to the $z$-axis.

$\mathrm{mm}$.

\subsection{Experiment}

The ratio between the measured magnetic field at the centre of the sample and the magnetic field at the centre of the exciting coil is $2.02 \mathrm{mT} / \mathrm{T}$. The maximal achievable magnitude and sweep rate of the applied field at the location of centre of the sample is $30 \mu \mathrm{T} / \mathrm{s}$ and $18.18 \mathrm{mT}$. The hollow cylindrical superconductor is a commercial tube from CAN Superconductors with dimensions shown in Figure 2. It is manufactured by pressing and sintering of polyerystalline $\mathrm{Bi}_{1.8} \mathrm{~Pb}_{0.26} \mathrm{Sr}_{2} \mathrm{Ca}_{2} \mathrm{Cu}_{3} \mathrm{O}_{10+x}$ (Bi-2223) powder. Given its polycrystalline nature, the properties of this sample are considered isotropic at the macroscopic scale.

We used a power law [27] $E(J)=E_{c}\left(J / J_{c}\right)^{n}$ to characterize the $E-J$ relation of the sample, where the threshold electric field $E_{c}$ is taken conventionally at $10^{-4} \mathrm{~V} / \mathrm{m}$ and $J_{c}$ is the critical current density. The critical exponent was determined previously by sweep-creep measurements [23] and found to be $n=30$.

The magnetic field dependence of the critical current for this material was approximated using Kim's law [28] $J_{c}(B)=J_{c 0} /\left(1+B / B_{1}\right)$. The two parameters of this law were determined experimentally by transport measurement: $J_{c 0}=6.26 \mathrm{MA} / \mathrm{m}^{2}$ and $B_{1}=8.9$ $\mathrm{mT}$ at $77 \mathrm{~K}$.

The shielding properties of this superconducting hollow cylinder were characterized in an axial homogeneous applied field configuration with a sweep rate of $0.1 \mathrm{mT} / \mathrm{s}$. The threshold induction $B_{\text {lim }}$ above which the shielding factor $S$ at the centre of the tube falls below 100 was found [29] to be $B_{\text {lim }}=6.75 \mathrm{mT}$ at $77 \mathrm{~K}$.

In order to measure the magnetic field distribution inside the superconducting hollow cylinder subjected to the field generated by the exciting coil, we use a bespoke 3- 


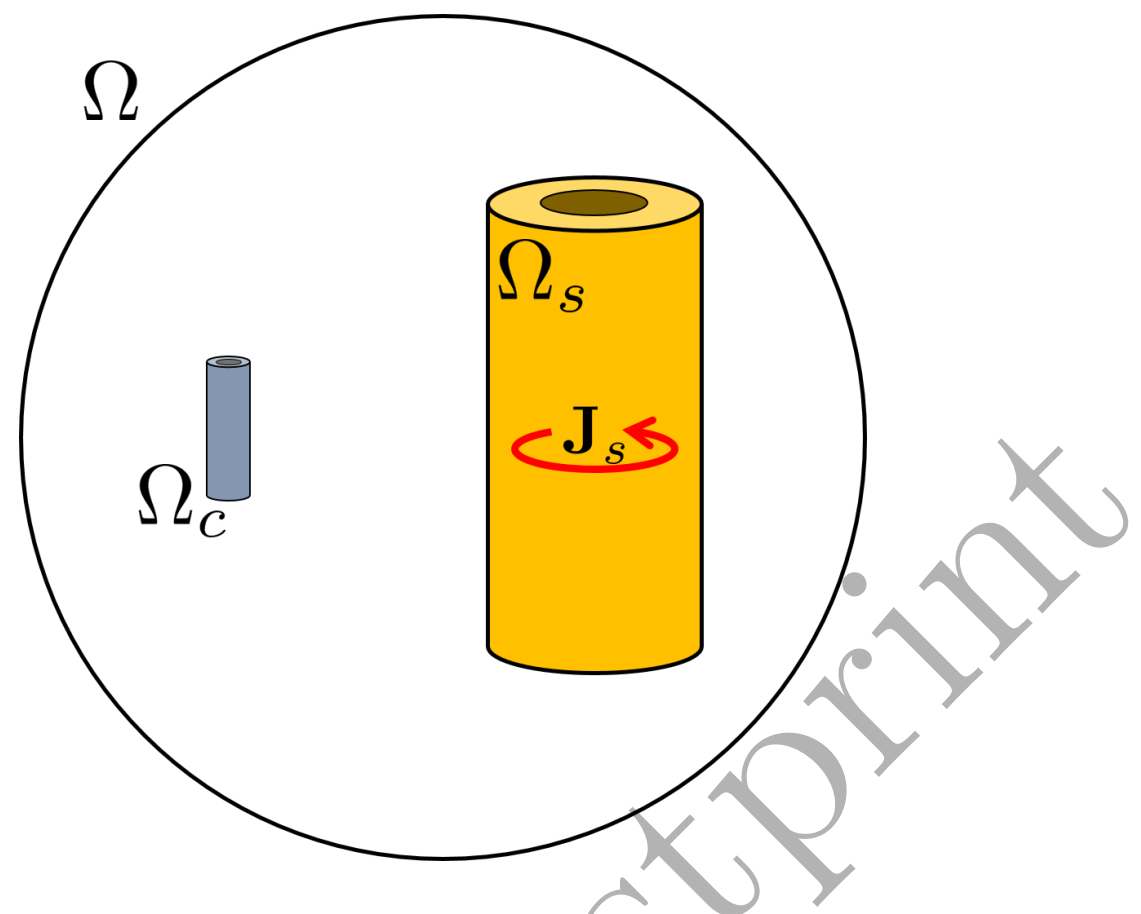

Figure 3. Diagram of the domains composing our model. $\Omega$ is the whole domain, $\Omega_{c}$ the superconductor and $\Omega_{s}$ the windings of the exciting coil in which a current density $J_{s}$ is imposed.

axis microdisplacement Hall probe system. It is composed of a high-linearity 3-axis cryogenic Hall probe mounted on three linear stages. This system was characterized in a homogeneous magnetic environment. It allows measurements of magnetic field as low as $0.1 \mathrm{mT}$ and can position the Hall probe in a $5 \mathrm{~cm}$ by $5 \mathrm{~cm}$ volume with an absolute on-axis accuracy of $290 \mu \mathrm{m}$. The maximum misalignment of the Hall probe was estimated to be less than 1 degree All experiments are carried out at liquid nitrogen temperature $(\mathrm{T}=77 \mathrm{~K})$

\section{Modelling}

In order to gain information on the magnetic field and shielding current distributions inside the superconductor, a full 3D finite element model based on a $\mathrm{H}$ formulation was developed.

The geometry we consider for numerical modelling is depicted on Figure 3, showing the superconducting shielding tube $\left(\Omega_{c}\right)$ and the exciting coil $\left(\Omega_{s}\right)$ in which a given current density is imposed to generate the magnetic field. Dimensions and positions are identical to those shown in Figure 2 .

As we want our model to allow us to investigate various inhomogeneous field configurations, we developed a full 3D model to compute the magnetic field around and in the superconducting hollow cylinder as well as the induced shielding currents. To do so, we opted for a $\mathrm{H}$ formulation.

Starting from magneto-quasi-static Maxwell's equations, we can obtain the following 
equation:

$$
\operatorname{curl}\left(\sigma^{-1} \operatorname{curl} \mathbf{H}\right)=-\mu_{0} \partial_{t} \mathbf{H},
$$

where $\sigma$ is the lectric conductivity and $\mu_{0}$ the magnetic permeability of vacuum. This equation constitutes the $\mathrm{H}$ formulation when coupled with appropriate boundary conditions. The unknowns are the three components of the magnetic field $\mathbf{H}$.

The modelling domain is divided in two regions depending on the value of the conductivity $\sigma$ : a conducting region, for which $\sigma \neq 0$, corresponding to the superconductor $\left(\Omega_{c}\right)$ and the insulating region where $\sigma=0$, which corresponds to the air and the source coil, i.e. the complementary of the superconductor $\left(\Omega_{c}^{C}\right)$. Indeed, the existing coil simply consists in an imposed current distribution in the air and it is therefore not modelled as a conducting material.

In so doing the number of unknowns can be reduced by taking advantage of information on the magnetic field. In the non-conducting region, we have no current, therefore we can model the magnetic field $\mathbf{H}$ as the gradient of an unknown scalar field $\phi$, i.e.

$$
\operatorname{curl} \mathbf{H}=\mathbf{0} \Leftrightarrow \mathbf{H}=-\operatorname{grad} \phi .
$$

This equation is valid as long as the non-conducting region is simply connected. In our case, however, we deal with a multiply connected domain because of the presence of the superconducting tube. In order to still be allowed to express the magnetic field as the gradient of a scalar field, therefore, a cut is introduced on one of the extremities of the tube [30] to make our region simply connected. This cut is a surface by which every closed curve in the non-conducting region that cannot be shrunk continuously to a point has to cross. The cut introduces a discontinuity in the scalar field $\phi$ which takes into account the total current embraced by the area of the closed curves.

Consequently, the basis functions used to model the magnetic field depend on the region. The magnetic field is defined on edge elements, using edge basis functions inside the superconductor, whereas, in the non-conducting region, it is defined on edge elements by using the gradient of nodal basis functions and the cut.

The magnetic field considered in our modelling is separated in two contributions:

$$
\mathbf{H}=\mathbf{H}_{\mathbf{s}}+\mathbf{H}_{\mathbf{r}} \text {. }
$$

The first term, the source magnetic field, $\mathbf{H}_{\mathbf{s}}$, is generated by the current imposed in the source coil that is such that

$$
\begin{array}{lll}
\operatorname{curl} \mathbf{H}_{\mathbf{s}}=\mathbf{J}_{\mathbf{s}} & \text { in } & \Omega_{s}, \\
\operatorname{curl} \mathbf{H}_{\mathbf{s}}=\mathbf{0} & \text { in } & \Omega_{s}^{C} .
\end{array}
$$

The second term, $\mathbf{H}_{\mathbf{r}}$, is the magnetic field generated by the shielding currents induced in the superconductor $\left(\Omega_{c}\right)$.

Superconducting properties are modelled by the power law for the $E-J$ relation and Kim's law for the magnetic dependence of the critical current $J_{c}$. It results in a nonlinear electric conductivity, as follows:

$$
\sigma^{-1}(J, H)=\frac{E_{c}}{J_{c 0}}\left(1+\frac{H}{H_{1}}\right)\left[\frac{J}{J_{c 0}}\left(1+\frac{H}{H_{1}}\right)\right]^{n-1} .
$$


The temporal resolution is based on an implicit Euler scheme. The nonlinearity of the resistivity in the superconductor is treated with the Newton-Raphson method.

The resolution scheme is a two steps process. First the source magnetic field, $\mathbf{H}_{\mathbf{s}}$, is computed inside the closed volume of the exciting coil (i.e. the coil itself and its bore) taking into account the imposed current density, $\mathbf{J}_{\mathbf{s}}$, inside the coil. In doing so, we impose that Ampere's law is verified with the imposed current density in order to know the source field:

$$
\operatorname{curl}\left(\partial_{t} \mathbf{H}_{\mathbf{s}}\right)=\partial_{t} \mathbf{J}_{\mathbf{s}}
$$

Second, the magnetic field induced by the shielding current in the superconductor is computed in the whole domain while imposing the solution of the source magnetic field inside the closed volume of the coil:

$$
\operatorname{curl}\left(\sigma^{-1} \operatorname{curl} \mathbf{H}_{\mathbf{r}}\right)+\mu_{0}\left(\partial_{t} \mathbf{H}_{\mathbf{s}}+\partial_{t} \mathbf{H}_{\mathbf{r}}\right)=0 .
$$

Inside the closed volume of the coil, a spanning tree technique [31] is used to gauge the source magnetic field $\mathbf{H}_{\mathbf{s}}$. For the reacting magnetic field, in the superconductor, we impose a spanning tree gauge, and in the air, as it is expressed as the gradient of a scalar field, we impose a Dirichlet boundary condition on the scalar field on the outer surface of the volume of the air: $\phi=0$. This boundary is sent to infinity by using a Jacobian transformation [32].

In the geometry shown in Figures 2 and 3 , the symmetry of the problem allows in principle only one half the domain to be modelled. It was chosen, however, to avoid using symmetry, so that the model could be used in totally asymmetrical situations, if needed. Taking into account the requirêment of a fine mesh for the superconductor, the whole domain is composed of around 400000 elements, of which roughly 24000 represent the superconducting tube (10 elements across the wall, 60 for the whole circumference, and 40 along its heighit).

We implement and solve this problem by using the finite element solver GetDP [33], which is an open-source solver developed by the Applied Computational Electromagnetism (A.C.E.) group of the University of Liege.

We use our model in two steps. First, we reproduce the situation corresponding as closely as possible to that of the experiments. For this purpose we use the superconducting parameters determined independently on previous experiments on the studied hollow cylinder. In a second step, we aim at analyzing the influence of parameters related to the inhomogeneity of the applied magnetic field. In this study, in Section 5, we choose to use a constant critical current instead of Kim's law.

\section{Results}

In this section, measurements and modelling results are presented and compared. It is recalled here that the term applied magnetic field refers to the magnetic field that would be measured at the centre of the hollow cylinder when it is in the normal state. 


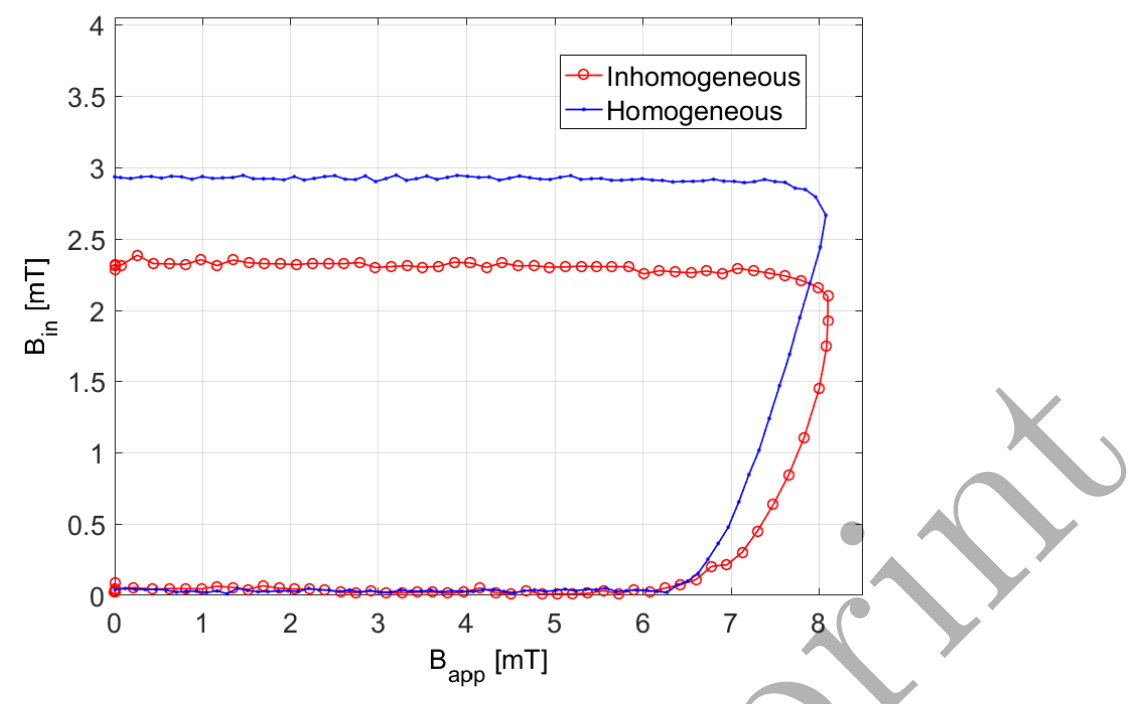

Figure 4. Measurements of the magnetic field at the centre of the superconducting tube when subjected to a homogeneous and an inhomogeneous magnetic field. In both case, the applied magnetic field at the eentre is the same $\left(B_{\max }=8.1 \mathrm{mT}\right.$ and $\left.\partial_{t} B_{a p p}=30 \mu \mathrm{T} / \mathrm{s}\right)$

\subsection{Comparison between homogeneous and inhomogeneous applied magnetic fields}

Figure 4 compares the magnetic flux density measured at the centre of the superconducting tube $\left(B_{i n}\right)$ when it is subjected to (i) the inhomogeneous field generated by the exciting coil shown in Fig. 2. and (ii) an axial homogeneous magnetic field. This comparison corresponds to the schematic illustration of flux lines shown on Fig. 1. In this experiment, the maximal applied magnetic field $B_{\max }$ at the centre of the tube, $8.1 \mathrm{mT}$, as well as its rate of variation, $30 \mu \mathrm{T} / \mathrm{s}$, are identical in both cases. The field is first increased from 0 to $B_{\max }$ and then switched off to zero at the same rate. As can be inferred from Fig. 44 the threshold induction $B_{l i m}$ for a shielding factor of 100 is measured to be $6.34 \mathrm{mT}$ with the inhomogeneous field and $6.4 \mathrm{mT}$ for the uniform axial field. In the present experiment, the value of $B_{\text {lim }}$ is slightly lower than the one mentioned in Section $2(6.75 \mathrm{mT})$ because the sweep rate of the applied field used here $(30 \mu \mathrm{T} / \mathrm{s})$ is smaller than the sweep rate used in previous experiments $(100 \mu \mathrm{T} / \mathrm{s})$. Knowing the critical exponent $\mathrm{n}$ of the superconductor, the ratio of the two $B_{l i m}$ values determined at different sweep rates can be estimated. Since $B_{\text {lim }}$ is proportional to the average critical current density $\langle J\rangle$ and the average electric field $\langle E\rangle$ proportional to the rate of the applied field $\partial_{t} B_{a p p}$ in a first order approximation [34], $B_{\text {lim }}$ is therefore proportional to $\left(\partial_{t} B_{a p p}\right)^{1 / n}$. Using the previously determined value $n=30$, the theoretical value of $B_{l i m}$ at $30 \mu \mathrm{T} / \mathrm{s}$ can be calculated to be $6.43 \mathrm{mT}$ which is close to the value we measured.

Another feature that can be observed from Figure 4 is that when the applied field is higher than the threshold field, the rate of increase of magnetic flux density inside the cylinder is larger for the homogeneous field than in for the inhomogeneous field. Due to 
the smoother increase in the inhomogeneous configuration, the remnant magnetic field is consequently lower.

This measurement result shown in Figure 4 allows us to point out one valuable difference between the two configurations. For a long tube in an axial homogeneous magnetic field, once a magnetic field is picked up by the sensor inside the cylinder, shielding currents are flowing everywhere in the superconductor and its magnetic response is maximal (as further shielding currents cannot appear). In the case of an inhomogeneous magnetic field, once a magnetic field is measured inside the shield, a portion of the superconductor might still be free of shielding currents and magnetic flux lines. We will see in the next section that is it indeed the case.

\subsection{Measurements in front, inside and at the back for three different magnetic field} amplitudes

In the inhomogeneous field configuration depicted in Figure 1, measurements of the magnetic field around and inside the superconducting hollow cylinder were carried out for three applied magnetic field amplitudes: $4 \mathrm{mT}\left(B<B_{l i m}\right), 6 \mathrm{mT}\left(B \approx B_{l i m}\right)$ and $8 \mathrm{mT}\left(B>B_{\text {lim }}\right)$. The measurements were performed along three lines parallel to the $z$-axis at different locations in the $y z$-plane. Three regions are distinguished: (a) the back of the tube (i.e. side not facing the coil), (b) inside the tube and (c) in front of the tube (i.e. the side facing the coil). Figure 5 and Figure 6 show measurements and modelling results of the amplitude and the orientation of the magnetic field at these three locations. They also display the applied magnetic field magnitude and orientation in the absence of cylinder for the $4 \mathrm{mT}$ case. Along the $z$-axis, $z=0 \mathrm{~mm}$ corresponds to the centre of the tube and $z=40 \mathrm{~mm}$ its extremity.

When the cylinder is in the normal state, Figure 5 shows that the magnitude of the applied magnetic field is indeed $4 \mathrm{mT}$ inside the superconductor (Fig. 5(b)) and almost constant along its height, i.e. from $z=0 \mathrm{~mm}$ to $z=40 \mathrm{~mm}$. The applied field decreases as the distance to the exciting coil increases, i.e. the field at the front is roughly 13 $\%$ higher than the one inside which, itself, is almost $14 \%$ higher than the one at the back. According to Figure 6, the field orientation, defined as the deviation angle $\zeta$ with respect to the $z$-axis, is $0^{\circ}$ in the median plane of the sample (i.e. the magnetic field is directed along the $z$-axis) and increases up to $\zeta=20^{\circ}$ along the three lines investigated. These results indicate that in this reference geometry, the superconducting cylinder is mainly subjected to a lateral field gradient , $\partial_{\rho} B_{z}$, where $\rho$ is the distance from the centre of the source coil in a cylindrical coordinates system, while the axial gradient of the field amplitude is almost zero. This lateral gradient is indissociably linked to a curvature of the flux lines observable through the appearance of a lateral component when one moves along $z$.

Now we turn to the amplitude of the magnetic induction measured when the hollow cylinder is in the superconducting state (plain symbols in Figure 6). We consider first the magnetic induction measured along the axis of the cylinder (Figure 5 (b)). For 


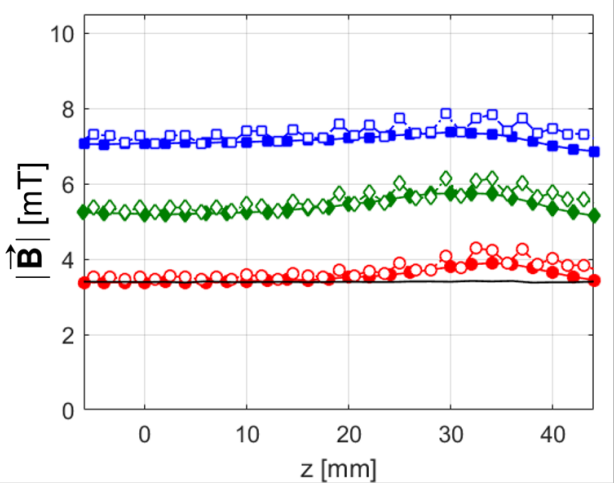

(a) Back

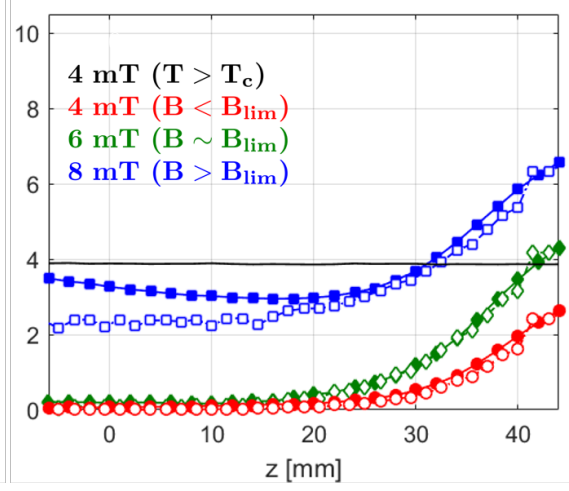

(b) Inside

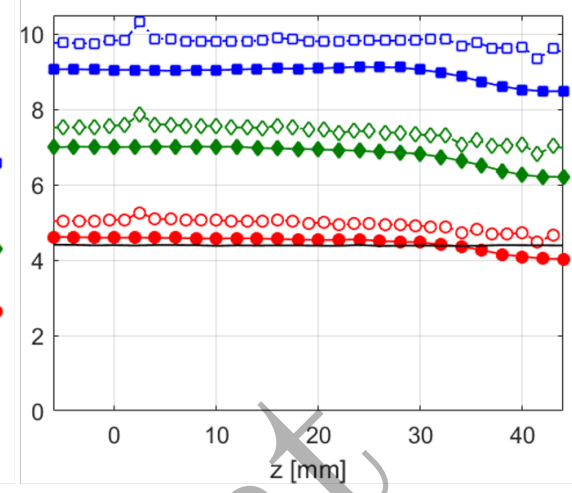

(c) Front

Figure 5. Measurements (full lines and solid symbols) and modelling results (dotted lines and open symbols) of the flux density modulus as a function of the position along the $z$-axis for three different applied magnetic field magnitude (4 $\mathrm{mT}$ (red circles), 6 $\mathrm{mT}$ (green diamonds) and $8 \mathrm{mT}$ (blue squares)) and at three different positions: (a) at the back of the superconductor, (b) inside the superconductor and (c) in front of the superconductor. The black lines corresponds to the magnetic field for $4 \mathrm{mT}$ when the superconductor is in the normal state $\left(T>T_{c}\right)$.

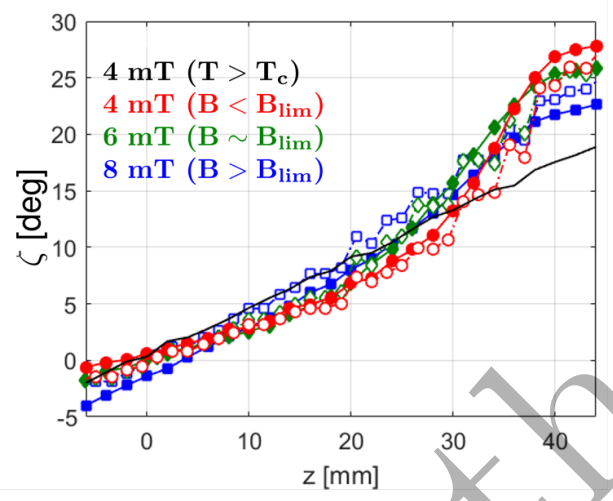

(a) Back

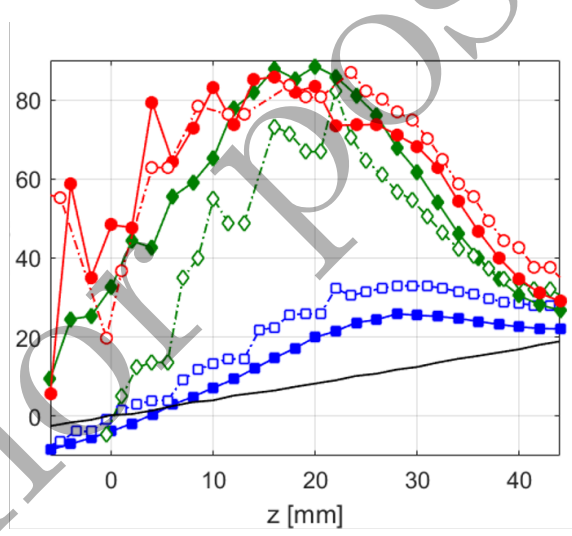

(b) Inside

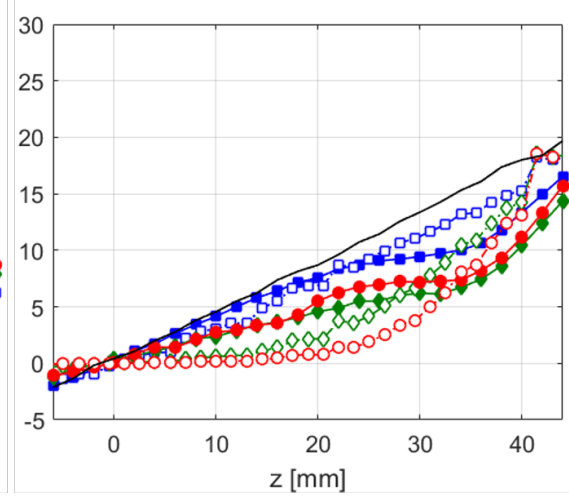

(c) Front

Figure 6. Measurements (full lines and solids symbols) and modelling results (dotted lines and open symbols) of the flux density orientation with respect to the $z$-axis as function of the position along the $z$-axis for three different applied magnetic field strengths (4 $\mathrm{mT}$ (red circles), $6 \mathrm{mT}$ (green diamonds) and $8 \mathrm{mT}$ (blue squares)) and at three different positions: (a) behind the superconductor, (b) inside the superconductor and (c) in front of the superconductor. The black line corresponds to the measurement of the magnetic field for $4 \mathrm{mT}$ at $T>T_{c}$.

applied magnetic field amplitudes of $4 \mathrm{mT}$ and $6 \mathrm{mT}$ (red and green symbols), the centre of the tube is shielded as no magnetic field is measured within the probe noise signal, in agreement with results shown in Figure 4. The measured magnetic induction increases near the extremity of the tube, due to the field penetration through the open ends [13]. When the applied field is $8 \mathrm{mT}$ (blue symbols), the magnetic field through the walls of the cylinder and the field distribution along the axis is globally moved $2 \mathrm{mT}$ above the distribution measured for $B_{a p p}=6 \mathrm{mT}$. In practice it can be seen that the 
magnetic induction along the central region $(z<25 \mathrm{~mm})$ is not constant but exhibits a slight minimum near $z=17 \mathrm{~mm}$ and not at the centre of the tube. This fact could be explained by a slight inhomogeneity of the superconducting properties of the cylinder and will not be discussed further.

Along an axis against the front of the tube (Figure 5(c)) the magnitude of the magnetic field is almost constant except near the extremity where it decreases. This is observed for all applied magnetic fields. For an applied field of $4 \mathrm{mT}$, one can observe that near the centre (for $z<30 \mathrm{~mm}$ ), the magnetic induction in the presence of the shield is slightly higher than without it.

At the back of the tube (Figure 5 (a)), we observe that the magnetic field magnitude reaches a slight maximum around $z=32 \mathrm{~mm}$ for all applied fields and in this region the magnetic field is higher than the applied field. Outside this region, its distribution is quite uniform along the $z$-axis near the centre $(z<15 \mathrm{~mm})$ and starts decreasing at the extremity of the tube $(z>40 \mathrm{~mm})$.

If we consider the modelled values of the field strength (open symbols in Figure 5), it can be seen that, except at the centre of the tube for the $8 \mathrm{mT}$ case (Figure 5 (b), blue lines), measurements and modelling results are in excellent agreement. It is recalled here that the superconducting properties of the sample-field-dependent critical current density and creep exponent - were obtained from previous independent experiments carried out under a uniform axial magnetic fields. At the front of the sample (Figure 5 (c)), we can notice that the modelling always gives field strengths slightly higher than the measurements but along the two other axes (inside and at the back of the cylinder) measurements and modelling are in remarkable agreement. The fact that modelling results are less smooth than the measurements is caused by the finite size of the mesh. In a 3D modelling, the number of elements should be kept moderate to allow for a reasonable computation time. The associated consequence is a relatively coarse mesh in the air. In the present case, it can be seen that the relatively coarse mesh outside the superconducting region does not hinder the modelling results from reproducing the principal features observed in the experiments.

We now investigate the magnetic field direction with respect to the axis of the tube. As can be observed in (Figure 6 (b)), it is inside the tube that the orientation of the flux density is affected the most (coloured symbols) compared to the data measured above $T_{c}$. For $4 \mathrm{mT}$ and $6 \mathrm{mT}$, the angle is roughly $30^{\circ}$ at the extremity and it increases toward the centre of the tube, up to reaching $90^{\circ}$ around $z \approx 20 \mathrm{~mm}$. In the central region of the cylinder $(z<20 \mathrm{~mm})$ both measurements and modelling results fluctuate since magnetic field magnitudes are globally below the noise level. For $8 \mathrm{mT}$ (blue symbols), the deviation from the axis is much smaller and does not reach $90^{\circ}$. It reaches a maximum around $z=30 \mathrm{~mm}$ and decreases toward the centre of the tube.

For the measurement and modelling results at the back of the tube (Figure 6 (a)), the maximum deviation from the orientation of the applied magnetic field takes place near the extremity of the cylinder. When moving from the extremity to the median plane ( $z$ decreasing), the angle with respect to the axial direction decreases as well and 
becomes smaller than the angle observed in the absence of shield (black line) for $z<28$ $\mathrm{mm}$. For all applied field strengths, the modelling is in excellent agreement with the measurements.

At the front of the tube (Figure 6 (c)), the deviation from the $z$-axis is always smaller than when no shield is there. The modelling results show that the magnetic field is mainly axial $(\zeta \approx 0)$ for $z<10 \mathrm{~mm}$ when the applied field is lower than $8 \mathrm{mT}$ and then increases when one moves towards the tube extremity ( $z$ increasing). The measurements exhibit rather a gradual increase, even for $z<10 \mathrm{~mm}$, as well as a slope change in the curve between $z=20 \mathrm{~mm}$ and $z=30 \mathrm{~mm}$. This could be explained by a slightly inhomogeneous critical current density, reminiscent to what was observed when analysing the modulus of the flux density in the central region. In spite of this difference, there is a fair overall agreement between the experiment and the modelling.

\subsection{Magnetic field distribution around the superconductor}

Figure 7 shows the flux density distribution in the $y z$-plane as the applied magnetic field increases from 0 to $8 \mathrm{mT}$. Given the symmetry of the problem, we only show the upper half of the superconductor. The magnetic field source is located at the right of the tube, as shown in Figure 2. At the centre of the superconducting tube, almost no flux density is observed up to $4.8 \mathrm{mT}$, in agreement with the previous graphs.

When examining the flux density in the tube walls, it can be observed that the zone where we find the maximal flux density is located near the extremity on the back side. In this region, the flux lines are almost perpendicular to the superconductor wall whereas they are almost parallel to the axis of the tube everywhere else. Logically, we observe that this region also corresponds to full penetration. This result shows that locally the whole thickness of the superconductor is penetrated and no more shielding currents can be induced while some zones of the superconductor are still current-free.

For steps (a) to (e), the flux lines enter through the tube open end but as the tube walls are not fully penetrated at the centre, these flux lines cannot exit through the other tube opening. They curve back to exit via the same opening and this leads to a magnetic field concentration at the upper back of the tube. At the step (f), the front wall is fully penetrated, even at the centre of the cylinder. As a consequence flux lines go through central region of the cylinder. The back wall, however, is not fully penetrated yet.

\subsection{Current density distribution inside the superconductor}

The current density distribution in the $y z$-plane inside the upper half of the superconductor for the situation detailed above is shown in Figure 8. Given the symmetry, the current direction in this plane is exclusively along the $x$-axis.

As the applied magnetic field increases, shielding currents are induced in the superconductor and they first appear on the outer surface of the sample.

Near the centre of the tube, the current density interface between current-free regions 


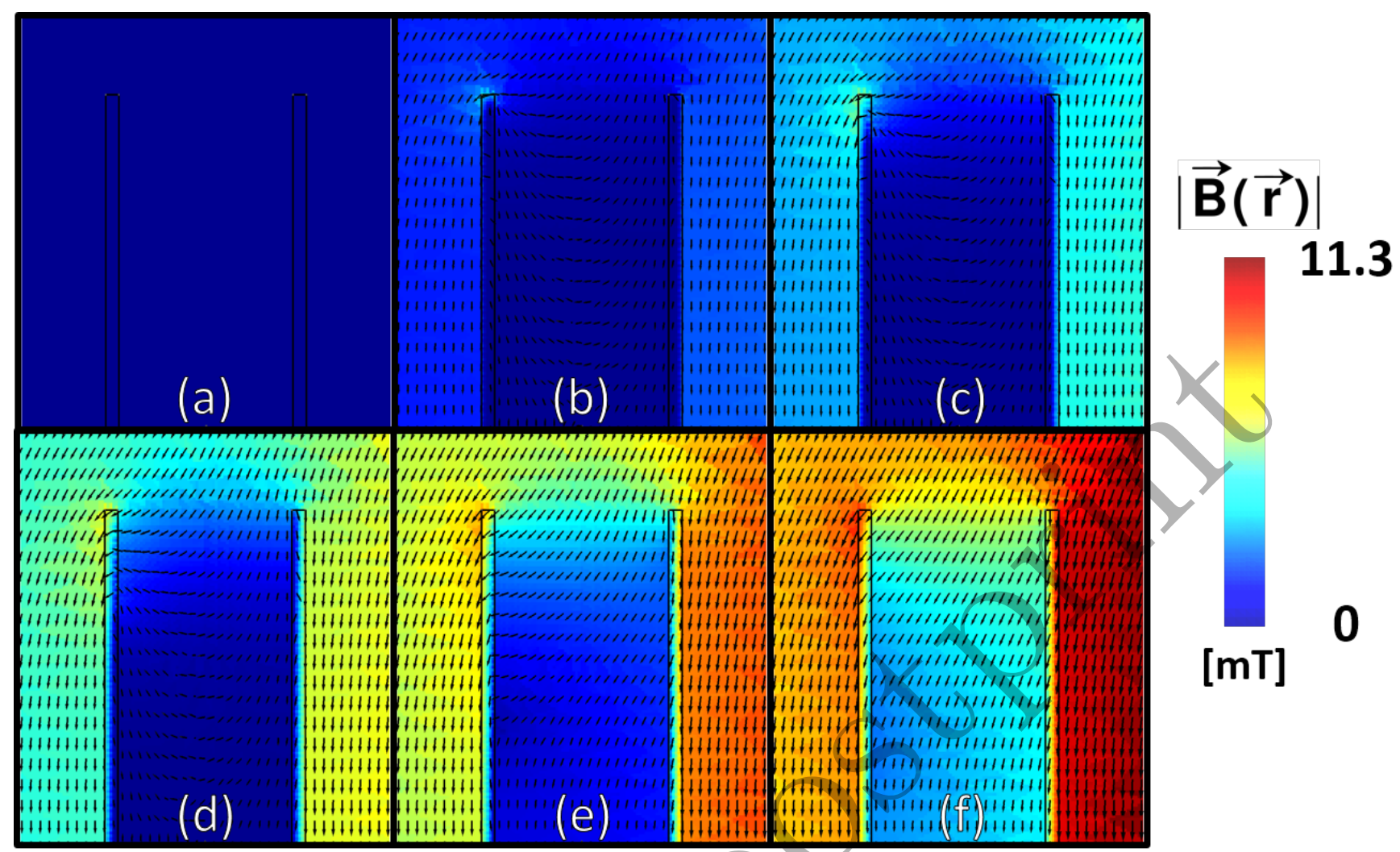

Figure 7. Calculated magnetic flux density distribution in $y z$-plane around the upper half of the superconductor when the applied magnetic field increases from 0 to $8 \mathrm{mT}$ with a rate of $30 \mu \mathrm{T} / \mathrm{s}((\mathrm{a}) 0 \mathrm{mT}$, (b) $1.6 \mathrm{mT}$, (c) $3.2 \mathrm{mT}$, (d) $4.8 \mathrm{mT}$, (e) $6.4 \mathrm{mT}$ and (f) $8 \mathrm{mT}$ ).

and non current-free regions is parallel to the the surface of the superconductor. We observe that this interface progresses faster on the front side than on the back side; the wall facing the magnetic field source is the first to be totally penetrated (Figure 8 (e)). When $8 \mathrm{mT}$ is reached, the superconductor is fully penetrated and exhibits no more current-free regions.

As shown in Figure 8 (c), near the extremity, the wall at the left is penetrated faster than the right wall although the latter is exposed to the highest magnetic field since it is nearer to the magnetic field source. In the wall region located at the front side of the superconductor, we observe currents flowing along the inner wall of the tube (blue color in Figure 8(d) and 8 (e)) in the same direction as in the wall region located at the back side. As the applied field increases, the front of these currents progresses inside the wall and propagates further toward the centre of the tube (Figure 8 (a) to (d)). Afterward, this front meets the one of the currents penetrating from the outer surface and the latter prevails on the former. In the final distribution (Figure 8 (f)), currents in the front side of the tube only flow in one direction, and the opposite currents that appeared along the inner surface have totally disappeared.

As we use a magnetic field dependent critical current density, the magnitude of the 


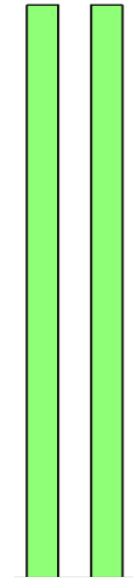

(a)

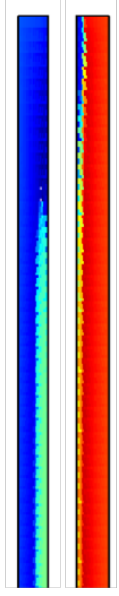

(d)

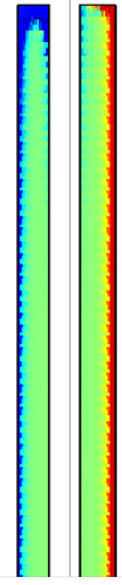

(b)

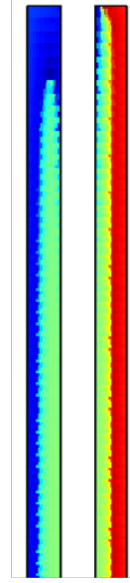

(c)

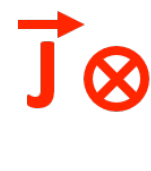

8

4.8

4.8

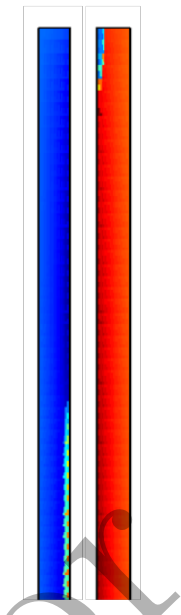

(e)

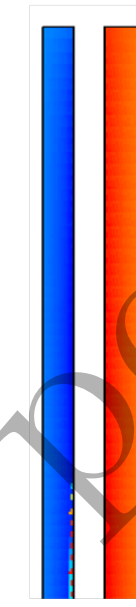

(f)

Figure 8. Simulated shielding current density distribution in the upper half of the superconductor when the applied magnetic field increases from 0 to $8 \mathrm{mT}$ with a rate of $30 \mu \mathrm{T} / \mathrm{s}((\mathrm{a}) 0 \mathrm{mT}$, (b) $1.6 \mathrm{mT}$, (c) $3.2 \mathrm{mT}$, (d) $4.8 \mathrm{mT}$, (e) $6.4 \mathrm{mT}$ and (f) $8 \mathrm{mT}$ ). The diameter between the two walls of the shield is not to scale on this figure. The source coil is on the right side of the superconducting tube.

current density is higher on the inner surface of the tube than on the outer one as the magnetic field is lower inside the tube.

To sum up, when a type-II hollow cylindrical superconductor is subjected to the fringe field of a nearby electromagnet, the main features can be summarized as follows: Given the geometry considered in our problem, the superconductor is mainly subjected to a transverse gradient of the applied field. This gradient is linked to a curvature of the applied field which is directed along the axis of the superconducting cylinder in its median plane and curves progressively to be directed at roughly $20^{\circ}$ with respect to the axis near the open ends. Unlike what happens in a uniform axial field, the penetration of flux lines is no longer axisymmetric. Interestingly, the local magnetic flux density 
experienced by the tube is shown to be larger on the back side near the extremities i.e. where the applied field is the smaller. This enhancement of the flux density in the back region of the tube is associated to a dissymetric curvature of the flux lines in the back and front regions of the tube. Experimental results of the magnetic flux density inside and against the tube walls (modulus and direction) were compared to 3D modelling results and found to be in excellent agreement. The agreement validates the model which is then used to visualize the flux density around the superconductor in more detail as well as the shielding current distribution inside the superconductor.

\section{Discussion}

In this section, we first discuss the validity of the reference geometry investigated to study fringe effects by comparing characteristics of the applied field in the absence of superconductor to general analytical expressions (Section 5.1). Then, we run our modelling tool using a constant critical current to investigate further flux front and penetration of the flux lines in the hollow superconducting cylinder. Following the idea of simplification of the results interpretation, we modelled the situation of a tube subjected to an oblique homogeneous magnetic field (Section 5.2p) as the current distribution in that situation is relevant for our interpretation of the results of Section 4. And finally, we used our model to investigate the impact of changing slightly the field distribution around the shield while keeping the applied magnetic field at the centre of the tube identical in all situations (Section 5.3.).

\subsection{Characteristics of the inhomogeneous applied field}

The results shown in Figure 6 (black lines) put into evidence two characteristics of the magnetic field applied to the superconducting cylinder: (i) a lateral gradient of the field, while the field strength along directions parallel to the axis of the cylinder is almost constant, and (ii) a linear variation of the angle of the field lines with respect to the axis of the cylinder. In order to evaluate the general character of this, we compare these features to analytical expressions available in the literature. For simplicity, we ignore the finite thickness of the exciting coil and consider a thin cylindrical solenoid of average radius $a$ and finite length $2 b$, as shown in Figure 9 .

The origin of coordinates is taken at the centre of the coil. To describe the magnetic field outside the coil, we consider a point $P$ located at distance $r$ at an angle $\theta$ from the $z$-axis (spherical coordinates), or use cylindrical coordinates $\rho=r \sin \theta$ and $z=r \cos \theta$. If the coil is made of $N$ turns of wire with constant current $I$, the sheet current in the solenoid is $K=N I / 2 b$, and its (dipolar) magnetic moment is $m=K\left(\pi a^{2} b\right)$. The flux density at the centre of the coil is axial and given by [35]

$$
B_{z}=\mu_{0} K \frac{b}{\sqrt{a^{2}+b^{2}}}
$$

In the literature, simple approached analytical expressions can be found to describe off-axis fields either for the internal field close to the axis [36] or the external field 


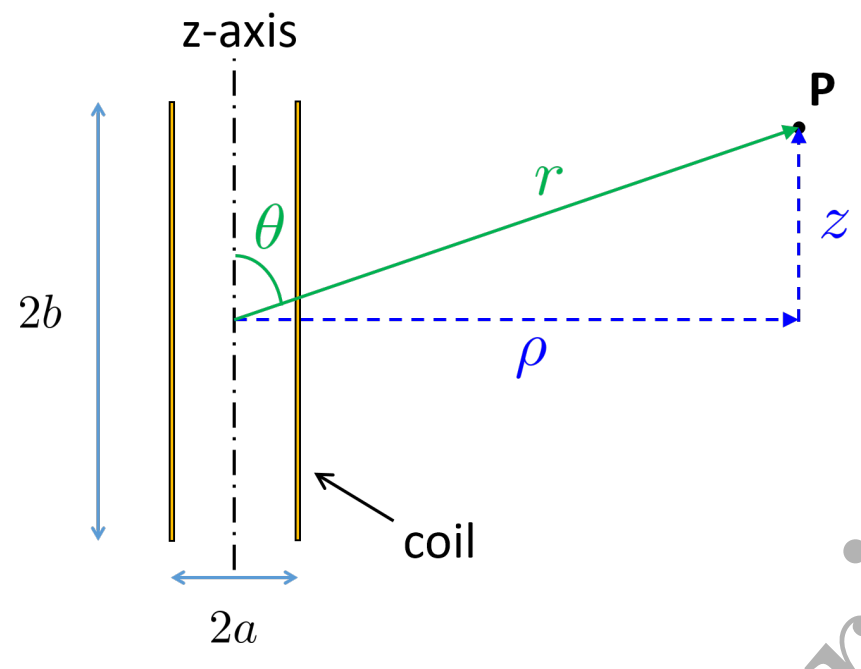

Figure 9. Thin cylindrical solenoid of radius $a$ and height $2 b$ with $N$ turns of wire carrying a constant current $I$.

just outside the solenoid [37, 38], i.e. at radial distances much smaller than its length $\rho \ll 2 b$. For the geometry studied in this work $\left(\rho \approx \rho_{0} \approx 8 a \approx 2 b\right.$, where $\rho_{0}$ denotes the average distance between the source coil and the superconductor), we can consider a far-field multipole expansion of the scalar potential $\phi$ in inverse powers of $r$, which can be expressed as [35]

$$
\phi(r, \theta)=\frac{K}{2}\left[\frac{A_{2}}{r^{2}} P_{1}(\cos \theta)+\frac{A_{4}}{r^{4}} P_{3}(\cos \theta)+\ldots\right],
$$

where the coefficients $A_{n}$ depend only the geometric dimensions $a$ and $b$ of the solenoid coil and $P_{n}$ denotes the Legendre polynomial of order $n$, i.e. $P_{1}(x)=x, P_{3}(x)=5 x^{3}-3 x$, etc. Using the analytical expression [35] of $A_{2}=a^{2} b$, the first term of Eq. (9) gives the scalar potential of a dipole, from which the radial $\left(B_{\rho}\right)$ and axial $\left(B_{z}\right)$ components of the flux density in cylindyical coordinates can be directly deduced. This gives

$$
\begin{aligned}
& \hat{B}_{\rho}=\frac{\mu_{0} m}{4 \pi}\left[\frac{3 \rho z}{\left(\rho^{2}+z^{2}\right)^{5 / 2}}\right], \\
& B_{z}=\frac{\mu_{0} m}{4 \pi}\left[\frac{2 z^{2}-\rho^{2}}{\left(\rho^{2}+z^{2}\right)^{5 / 2}}\right] .
\end{aligned}
$$

The corresponding modulus $B=|\mathbf{B}|=\sqrt{B_{\rho}^{2}+B_{z}^{2}}$ and deviation angle with respect to the $z$-axis $\zeta=\tan ^{-1} \frac{B_{\theta}}{B_{z}}$ as well as their approximation for region satisfying $z \ll \rho$ are given by

$$
\begin{aligned}
& B=\frac{\mu_{0} m}{4 \pi} \frac{\sqrt{4 z^{4}+5 \rho^{2} z^{2}+\rho^{4}}}{\left(\rho^{2}+z^{2}\right)^{5 / 2}} \approx \frac{\mu_{0} m}{4 \pi \rho^{3}}\left[1-3\left(\frac{z}{\rho}\right)^{4}\right], \\
& \zeta=\tan ^{-1}\left(\frac{3 \rho z}{2 z^{2}-\rho}\right) \approx-3\left(\frac{z}{\rho}\right) .
\end{aligned}
$$

Importantly, the modelled and measured values of $B$ and $\zeta$ shown in Figure 5 and Figure 6 in the absence of superconductor are in agreement with the above expression. First 
the field strength along directions $z$ at constant $\rho$ is almost constant if the condition $(z / \rho) \ll 1$ holds, and second, the angle of the field lines with respect to the axis of the cylinder varies linearly along $z$. At the extremity of the cylinder, in particular, one has $(z / \rho)=\left[(h / 2) / \rho_{0}\right] \approx 0.114$, and the theoretical angle predicted by the approximate Eq. $(11 b)$ is thus $0.342 \mathrm{rad}=19.6^{\circ}$, in agreement with the data plotted in Figure 6 .

Interestingly, if the field lines at the location of the sample can be replaced by those of a dipole having the same magnetic moment, this means that the relevant parameter of the exciting coil is its dipolar magnetic moment, independent of its aspect ratio $(b / a)$. The validity of the dipolar approximation, however, can be checked by estimating the magnitude of the following terms appearing in Eq. (9) which depends of the aspect ratio $(b / a)$. As an example, the coefficient $A_{4}$ appearing in Eq. (9) can be calculated [35] to be $A_{4}=a^{2} b^{3}-(3 / 4) a^{4} b$. Hence the second term of the scalar potential (octupolar term) is

$$
\phi_{\text {oct }}=\frac{K}{2} a^{2} b\left(b^{2}-\frac{3}{4} a^{2}\right)\left(\frac{1}{2 r^{4}}\right)\left(5 \cos ^{3} \theta-3 \cos \theta\right) .
$$

The consequence is that at an average distance $r$ from the coil, and neglecting the $\theta$ dependence, the importance of this term depends globally on the dimensionless factor $\left[b^{2}-(3 / 4) a^{2}\right] / r^{2}$. Therefore the aspect ratio of the coil is not crucial in the present geometry, but is expected to play role as soon as $\left[b^{2}-(3 / 4) a^{2}\right] / r^{2}>1$. Modelling results of a hollow cylinder subjected to the field generated by exciting coils of various aspect ratios will be presented in Section 5.3 .

From the above analysis, the major conclusion to be drawn is that the characteristics of the magnetic field applied to the sample in the geometry of Figure 2 are very close to that of a dipole placed at the centre of the source coil, even though the average distance $\rho_{0}$ between the coil and the superconducting cylinder is not very large compared to the coil dimensions, i.e. here one has $\rho_{0} \approx 2.5 b$. It is therefore expected that the qualitative features reported in this work still hold true in cases where the dipole approximation still holds, e.g. at larger distances from the source coil if all other dimensions are the same. This latter configuration corresponds to the one encountered in many practical applications.

\subsection{Comparison between an inhomogeneous field and an oblique homogeneous field}

Having examined the characteristics of the inhomogeneous applied field and its unavoidable curvature, we now turn to determining the magnetic field penetration and the distribution of shielding currents.

As mentioned in Section 1, for the case of a magnetic shield subjected to inhomogeneous magnetic fields, shielding currents paths cannot be easily predetermined if the applied magnetic field is not applied along a symmetry axis or a symmetry plane of the superconductor shield. To illustrate this fact, consider the simple case of an infinite cylindrical superconductor in the Meissner state subjected to an oblique magnetic field. It can be shown that all shielding currents flow parallel to a plane, which, contrary 
to what one could have naively guessed, is not perpendicular to the direction of the applied field. This behavior results from a difference in the demagnetisation factors associated with the axial and transverse components of the applied field. Thus, the loss of symmetry between the applied field and the superconductor, together with end effects, results in complex shielding currents paths.

To proceed, in order to simplify the analysis and also determine what field characteristics are predominant regarding the flux penetration, we consider the situations where a superconducting cylinder is subjected to either (i) an inhomogeneous curved field or (ii) a homogeneous oblique field.

This is done by modelling the superconductor under either $J_{c}(B)$ or a constant $J_{c}$, in order to point out the contribution of field dependence of the critical current density effect. We use a constant critical current of $J_{c 0}=2.5 \mathrm{MA} / \mathrm{m}^{2}$ which corresponds to the critical current in $J_{c}(B)$ for $B=6 \mathrm{mT}$, i.e. roughly the $J_{c}$ value when the field lines penetrate inside the cylinder.

Figure 10 compares the current density distribution in the superconductor in the cases of an inhomogeneous magnetic field and an oblique homogeneous magnetic field for either a field-dependant or a constant critical current density. In all situations, the applied field at the centre of the tube is identical and increasing from 0 to $8.8 \mathrm{mT}$ with a sweep rate of $30 \mu \mathrm{T} / \mathrm{s}$.

Figure 10 (a) corresponds to the current density distribution in the superconductor as shown on Figure 8 in the previous Section while (c) corresponds to the situation with a constant $J_{c}$. The full penetration(is reached earlier with a constant $J_{c}$ as the current density is lower than $J_{c 0}$. These currents in the opposite direction on the inner upper front side of the tube are observed in all investigated cases. The results plotted in Figure 10 (a) and Figure 10 (c) show that the magnetic flux penetration in the superconductor with a constant $J_{c}$ behaves qualitatively the same way as with a field-dependant $J_{c}$, but with sharper front ends. Therefore results in constant $J_{c}$ can be used to apprehend the penetration mechanisms.

Figure 10 (b) and Figure 10 (d) show results for the case of an oblique field with an angle of $15^{\circ}$. We chose that value close to the applied field orientation in the inhomogeneous case.

The current density distribution in the oblique field configuration situation is depicted in Figure 10 (b) in the case of a field-dependent $J_{c}$ and (d) in the case of a constant $J_{c}$. We observe that current flowing in the opposite direction on the inner surface in the upper right side appears. The difference is that these currents are still present when the tube is fully penetrated while they disappear in the case of the inhomogeneous field.

It is of interest to note that what is common in both situations, at the location where we observe those opposite currents is that flux lines on both sides of the wall are directed along the same direction. The schematic representation of the flux lines around the extremity of the tube when subjected to the inhomogeneous magnetic field is depicted on Figure 11 (a). The front side of the tube experiences a field which is only directed in one direction while the back side experiences a field with opposite direction on both 


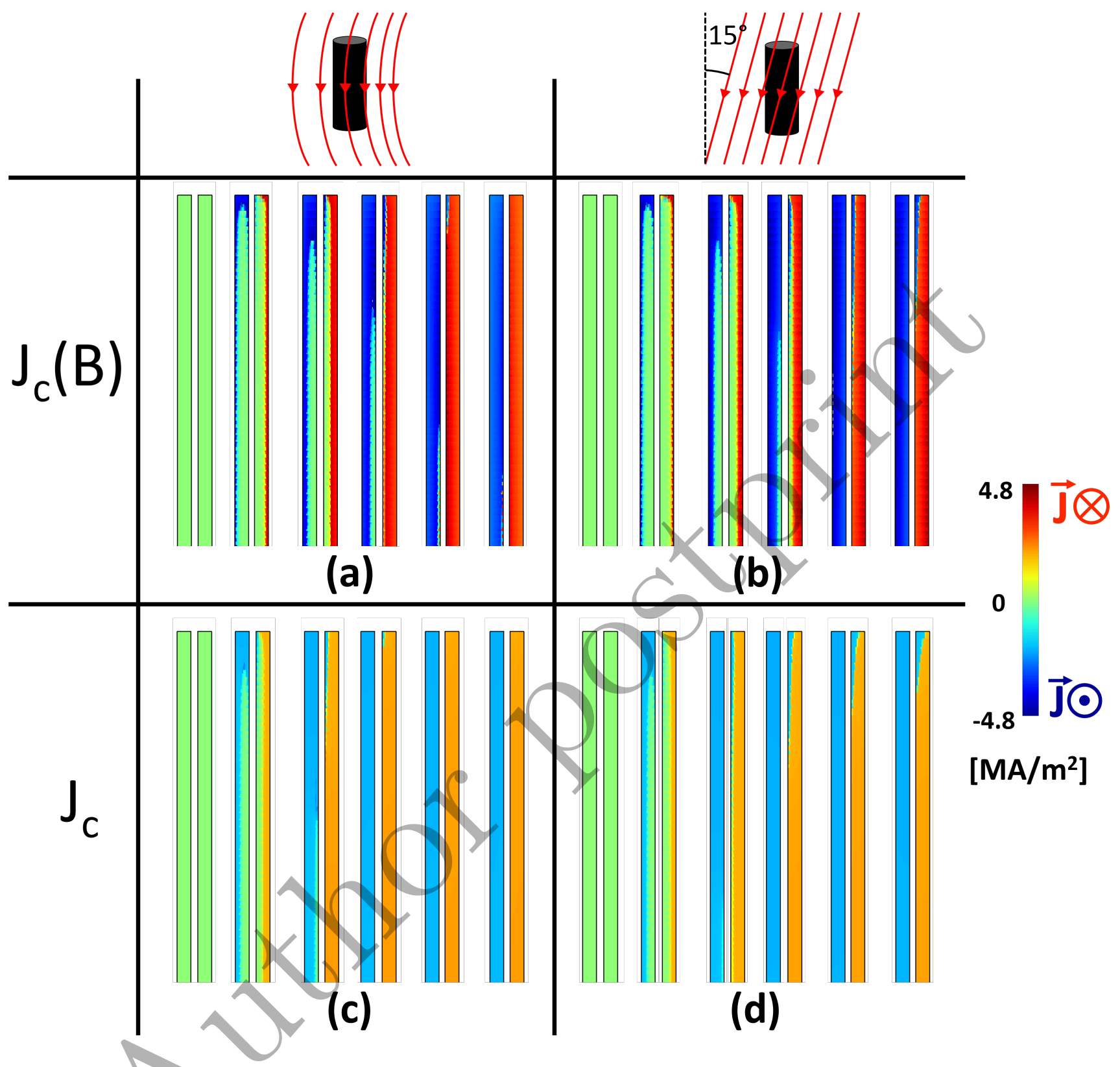

Figure 10. Current density distributions in the upper half of the superconductor for four cases. (a) and (b) have field-dependant critical $J_{c}$ while (c) and (d) have constant $J_{c}$. For (a) and (c) the applied field is inhomogeneous and generated by the exciting coil and for (b) and (d) it is homogeneous and oblique with a $15^{\circ}$ angle. In all case the applied field at the centre of the tube increases linearly from 0 to $8.8 \mathrm{mT}$ at $30 \mu \mathrm{T} / \mathrm{s}$ shown here by steps of $1.76 \mathrm{mT}$. 


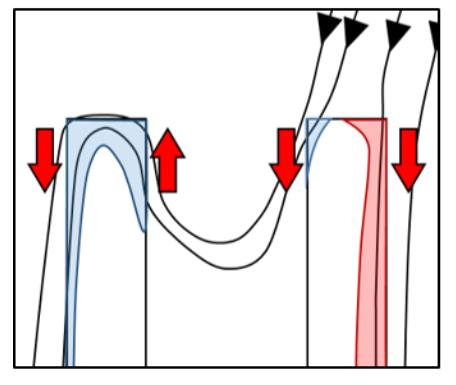

(a)

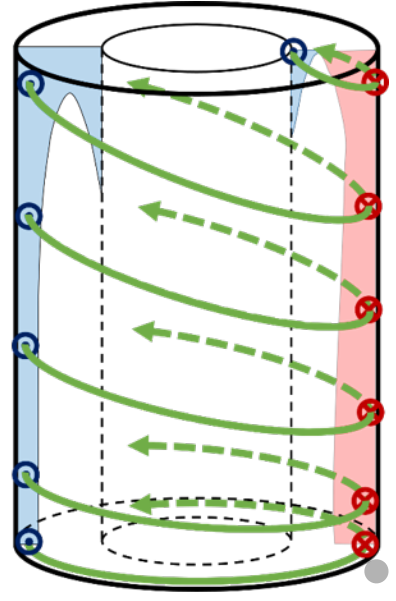

(b)

Figure 11. Schematic representations of (a) flux lines around the extremity of the tube and (b) the shielding current induced in the upper half of the superconducting tube in the case of the inhomogeneous magnetic field.

sides.

Therefore, the currents in the opposite direction appear because the shielding currents aim at reducing the flux density in the wall of the superconductor itself. Figure 11 (b) represents the schematic current distribution in the shield subjected to an inhomogeneous magnetic field. From the point of view of the upper front region of the tube, there is no way to have shielding currents flowing around the hole of the tube. The consequence is that there are current loops closing only on one side of the hole in the tube. These currents are not generating a magnetic field that opposes the flux that enters in the hole.

These results allow us to conclude that the main component characterizing the shielding current distribution inside the sample is due to the orientation of the magnetic field more than its lateral gradient (i.e. its magnitude variation along $\rho$ ).

\subsection{Curvature of the applied magnetic field and the aspect ratio of the source coil}

It was shown in Section 5.1 that the magnetic field applied to the superconductor in the reference geometry depicted in Figure 2 depends mainly on the magnetic moment of the coil, i.e. the dipolar approximation holds. The aspect ratio $(b / a)$ of the coil is expected to play a secondary role. In order to investigate this, the numerical model was used with different aspect ratios. In order to compare the results, we choose to keep the magnetic field experienced by the superconductor at its centre identical in all situations by adapting the current imposed in the exciting coil. The aspect ratio of the magnetic source is changed by varying the height of the coil while keeping its radius constant. By doing so, we mainly change the curvature of the magnetic flux lines near the superconductor while keeping constant the magnitude of the field experienced by 
the sample. We expect the effect of the change of curvature to be more pronounced near the extremities of the tube than near the centre. The induced field orientation change cannot be predicted by taking only into account the dipolar component of the multipole expansion as stated by Eq. (11b).

We tested three different aspect ratios: twice (h21), half (h12) and a quarter (h14) of the initial height of the exciting coil.

The results plotted in Figure 6 pointed out that a magnetic field concentration occurs around the superconducting shield. To evaluate this effect, we compute a concentration factor $\alpha$, which is the ratio of the magnetic field obtained when the superconductor shields and that measured at the same point if the superconductor was removed.

The magnetic field concentration factor for the four configurations discussed above is depicted on Figure 12 at four different locations around the shield, at a distance of 0.1 $\mathrm{mm}$ from the surface: (a) and (c) are located at the back of the tube, respectively at the upper side and the centre of the tube while (b) and (d) are on the side facing the coil. The greatest concentration factor is found at point (a), for the coil with the lowest aspect ratio (h14), where it almost reaches 2 for small applied field magnitude. As the applied magnetic field increases, $\alpha$ decreases tending towards the value 1 . When we change the aspect ratio of the source coil, we observe a-non-linear variation of $\alpha$ but in all situations the behaviour is identical. The lowest concentration factor is obtained at point (b). At this point, the concentration factor presents a minimum, the higher the aspect ratio of the coil, the higher the applied field for which this minimum is reached. At low field amplitudes, the factor $\alpha$ decreases. It is higher than 1 only for h21. For both points at half the height of the tube, when the field magnitude is higher than $6 \mathrm{mT}$, the concentration factor is the same for all aspect ratio, higher than 1 and decreasing. At the back of the tube, at low field magnitude, the factor is less than one in all cases. The lower the aspect ratio, the lower $\alpha$. As the field increases, the concentration factor increases as well and becomes greater than 1 until reaching a maximum. At the front of the tube, there is always a concentration, i.e $\alpha>1$. It is higher when the aspect ratio of the coil is the smaller. At first, the factor decreases linearly until the applied field reaches $3.5 \mathrm{mT}$, at which point, it begins to drop faster towards 1 . This kink coincides with the full penetration of the wall of the tube facing the coil.

These results clearly show that near the ends of the tube on the side not facing the source coil is the location where the shield experiences the highest concentration factor. This is to put in parallel with the results we presented in Section 4 showing that this location correspond to the first portion of the shield to be totally penetrated by the applied field.

In summary, we showed that the magnetic field experienced by the superconductor in the situation we investigate can be approximated as the field generated by an equivalent magnetic dipole at the centre of the coil. We discussed the shielding current distribution in the superconductor and pointed out how the inhomogeneity of the field, and mainly the field orientation, affected it and the magnetic response of the shield. We 


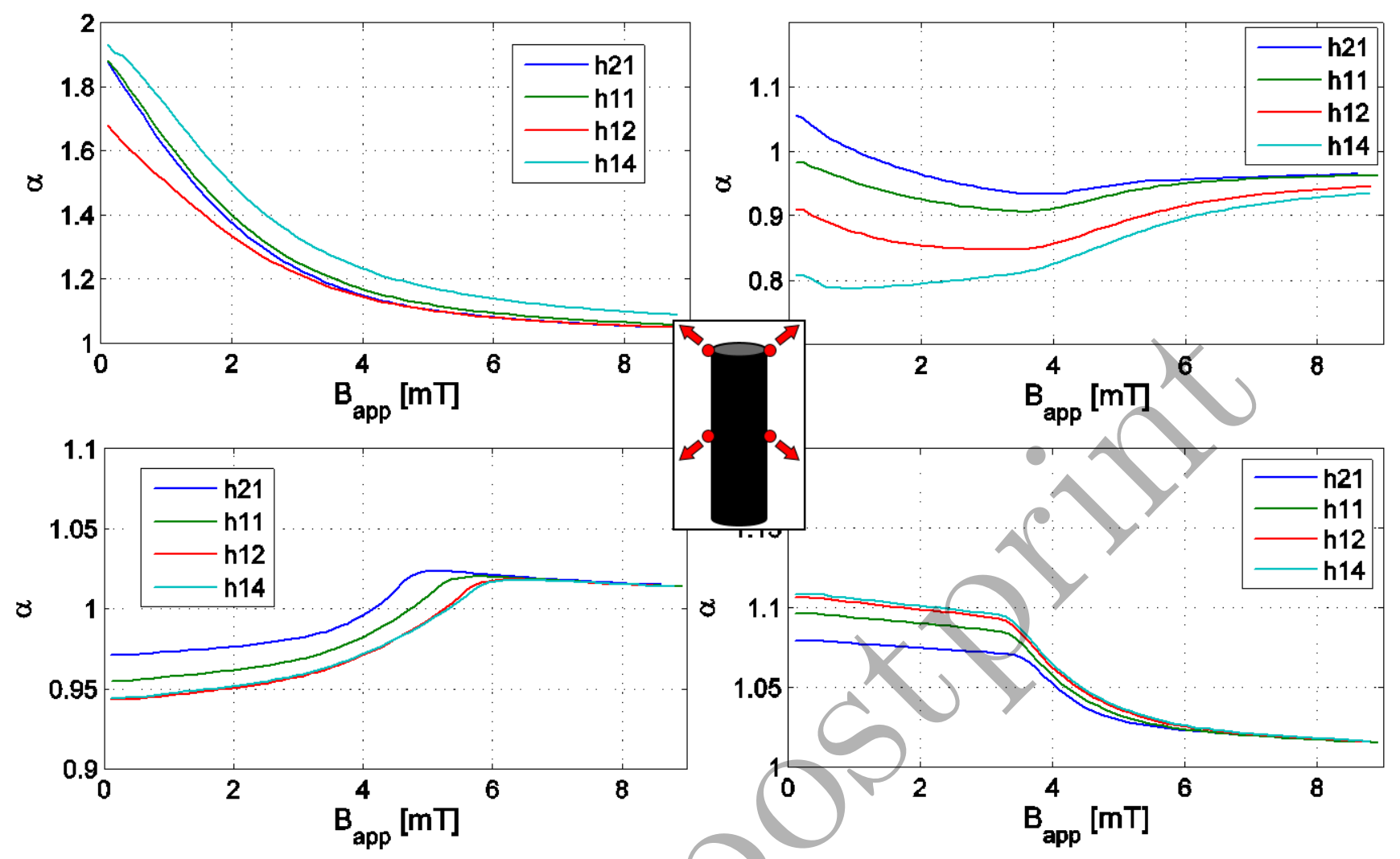

Figure 12. Magnetic concentration factor for different aspect ratio of the source coil (h21,h11,h12,h14) for a maximal applied magnetic field of $8.8 \mathrm{mT}$, at four points around the superconductor: (a) near the upper corner on the side opposite to the source coil, (b) same but on the side facing the coil, (c) on the opposite side at the centre of the tube and (d) identical but on the other side.

compared our situation to that of the case of a homogeneous oblique field and found that the current distribution is mainly due to the applied field orientation. Finally, we discussed the magnetic field concentration around the shield when modifying the field lines curvature around it and pointed out the weak spots in the shield.

\section{Conclusion}

In this work, the magnetic shielding properties of a bulk type-II superconducting tube were investigated in the case of an inhomogeneous magnetic field generated by a nearby solenoidal coil. This situation is representative of the magnetic field configurations that are encountered in practical applications e.g. a sensitive equipment which has to be placed near a high magnetic field electromagnet. Unlike in studies carried out under a homogeneous field, the investigated configuration leads to some difficulties for interpreting the reaction of the superconductor as it experiences a field whose amplitude and orientation are position-dependent. In agreement with our previous work, the applied magnetic field is defined as the magnetic field that would be measured at the centre of 
the shield when in its normal state. We also discussed the features characterizing the inhomogeneity of the magnetic field in terms of magnitude and its curvature. It was shown that the solenoidal magnetic field source considered here (aspect ratio: length / diameter $\approx 3.2$ ) can be approximated by an equivalent magnetic dipole when the average distance between the magnetic field source and the superconductor is of the order of 1.2 times the length of the solenoid.

We experimentally characterized the magnetic response of our bulk high temperature superconducting tube when subjected to this inhomogeneous magnetic field thanks to a bespoke microdisplacement system allowing measurements of the magnetie field distribution nearby a sample at $77 \mathrm{~K}$. To obtain information on the shielding currents and magnetic field distributions in the superconductor, we developed a full 3D finite element model based on a $\mathrm{H}$ formulation. Using the magnetic field dependent critical current and the creep exponent obtained from previous independent experiments carried out under homogeneous magnetic fields, our model computed the magnetic field distribution around the superconducting tube. These results were found to be in very good qualitative and quantitative agreement with the experimental measurements.

The mechanisms of magnetic field penetration and the shielding current distribution in a tubular type-II superconducting sample was investigated using our model. We pointed out that several zones of the superconducting walls are subjected to magnetic field concentration. The ones experiencing the highest magnetic field concentration and which are the first to be fully penetrated are located at the tube extremities on the side not facing the magnetic field source. In order to simplify the interpretation of the current density distributions in the superconductor, we modified our model to use a constant critical current density. We also studied, in that case, the situation of a superconducting tubesubjected to an oblique magnetic field. We showed that the current density distribution near the extremities of the tube behaves in the same way in that situation. This helped us clarifying the current density distribution inside the shield. The emerging picture is that induced current loops in some parts of the tube flow as to prevent vortices from entering in the superconductor wall, without protecting the inside of the tube. The situation is in contrast with that of a tube subjected to an axial field in which field lines approach axially and azimuthal induced currents oppose the entry of flux lines in both the wall and the hollow part of the tube. We investigated the impact of the inhomogeneity of the magnetic field, mainly the flux lines curvature, by changing the shape of the source coil while keeping constant the applied field at the centre of the shield. We observed that the highest magnetic field concentration around the shield for each case is at the extremity not facing the coil.

The results obtained in this work show that superconducting magnetic shields, even of simple shape, subjected to inhomogeneous magnetic field configurations are nontrivial and require additional considerations for design because the shielding current 
distribution cannot be predicted intuitively in a straightforward manner and magnetic field concentration might occur and deteriorate the shielding properties.

\section{Acknowledgments}

We thank the Communauté Française de Belgique under reference ARC 11/16-03 for cryofluids and equipment grants. K. Hogan is holder of a research grant from the Fonds pour la formation la Recherche dans l'Industrie et dans l'Agriculture (FRIA).

\section{References}

[1] Bean C P 1964 Reviews of Modern Physics 36 31-39

[2] Bhagwat K and Chaddah P 1989 Pramana 33 521-540

[3] Clem J R and Sanchez A 1994 Physical Review B 50 9355-9362

[4] Brandt E H 1994 Physical Review B 50 4034-4050

[5] Brandt E H 1998 Physical Review B 58 6506-6522

[6] Pavese F 1998 Magnetic shielding Handbook of Applied Superconductivity, Volume 2 vol 53 ed Seeber B (Taylor \& Francis) pp 1461-1483

[7] Fagnard J F, Elschner S, Bock J, Dirickx M, Vanderheyden B and Vanderbemden P 2010 Superconductor Science and Technology 23095012

[8] Rabbers J J, Oomen M P, Bassani E, Ripamonti G and Giunchi G 2010 Superconductor Science and Technology 23125003

[9] Ohta H, Koike A, Hoshino K, Kotaka H, Sudoh E, Kato K, Takahara H, Uchikawa Y, Shinada K, Takahata M, Yamada Y and Matsui T 1993 IEEE Transactions on Appiled Superconductivity 3 $1953-1956$

[10] Gomory F, Solovyov M, Souc J, Navau C, Prat-Camps J and Sanchez A 2012 Science 3351466 1468

[11] Martin F, Lorant S and Toner W 1972 Nuclear Instruments and Methods 103 503-514

[12] Symko O G, Yeh W J, Zheng D J and Kulkarni S 1989 Journal of Applied Physics 652142

[13] Denis S, Dusoulier L, Dirickx M, Vanderbemden P, Cloots R, Ausloos M and Vanderheyden B 2007 Superconductor Science and Technology 20 192-201

[14] Arpaia P, Ballarino A, Giunchi G and Montenero G 2014 Journal of Instrumentation 9 P04020$\mathrm{P} 04020$

[15] Haseyama S, Fujinaka N, Yoshizawa S and Nakane H 2001 Physica C: Superconductivity and its Applications 354 437-440

[16] Wéra L, Fagnard J F, Levin G a, Vanderheyden B and Vanderbemden P 2015 Superconductor Science and Technology $\mathbf{2 8} 074001$

[17] Kvitkovic J, Hatwar R and Pamidi S 2016 IEEE Transactions on Applied Superconductivity 26 $1-5$

[18] Tomkow L, Ciszek M and Chorowski M 2016 IEEE Transactions on Applied Superconductivity 26 $1-4$

[19] Giunchi G, Bassani E, Hassel J, Helistö P and Penttilä J 2010 MGB2 cylindrical cup for magnetic shielding in pulse tube cryostat AIP Conference Proceedings vol 1218 pp 1636-1640

[20] Gozzelino L, Gerbaldo R, Ghigo G, Laviano F and Truccato M 2017 Journal of Superconductivity and Novel Magnetism 30 749-756

[21] Kostrov E, Demikhov E, Bagdinov A, Demikhov T, Lysenko V and Tysyachnykh Y 2016 IEEE Transactions on Applied Superconductivity 26 1-5

[22] Hofman M B M, Kuijer J P a, de Ridder J W, Perk L R and Verdaasdonk R M 2013 Medical physics $\mathbf{4 0} 012303$ 
[23] Hogan K, Fagnard J F, Wéra L, Vanderheyden B and Vanderbemden P 2015 Superconductor Science and Technology $\mathbf{2 8} 035011$

[24] Hull J R 2000 Superconductor Science and Technology 13 R1-R15

[25] Werfel F N, Floegel-Delor U, Rothfeld R, Riedel T, Goebel B, Wippich D and Schirrmeister P 2012 Superconductor Science and Technology 25014007

[26] Callen J D 2003 Fundamentals of Plasma Physics (Unpublished)

[27] Rhyner J 1993 Physica C: Superconductivity 212 292-300

[28] Kim Y B, Hempstead C F and Strnad A R 1962 Physical Review Letters 9 306-309

[29] Wéra L, Fagnard J f, Hogan K, Vanderheyden B and Vanderbemden P 2016 Magnetic Shielding with Bulk High Temperature Superconductors : Improvement of the Shielded Volume in Hollow Cylinders Superconductivity: Applications Today and Tomorrow ed Muralidhar M (Nova Science) pp 95-114

[30] Dular P, Legros W and Nicolet A 1998 IEEE Transactions on Magnetics 34 3078-3081

[31] Dular P, Henrotte F, Robert F, Genon A and Legros W 1997 IEEE Transactions on Magnetics 33 1398-1401

[32] Henrotte F, Meys B, Hedia H, Dular P and Legros W 1999 IEEE Transactions on Magnetics 35 $1434-1437$

[33] Dular P, Geuzaine C, Henrotte F and Legros W 1998 IEEE Transactions on Magnetics 34 33953398

[34] Fagnard J F, Dirickx M, Ausloos M, Lousberg G, Vanderheyden B and Vanderbemden P 2009 Superconductor Science and Technology 22105002

[35] Durand E 1968 Magnetostatique (Paris: Masson et/Cie.)

[36] Muniz S R, Bagnato V S and Bhattacharya M 2015 American Journal of Physics 83 513-517

[37] Farley J and Price R H 2001 American Journal of Physics 69 751-754

[38] Labinac V, Erceg N and Kotnik-Karuza D 2006 American Journal of Physics 74 621-627

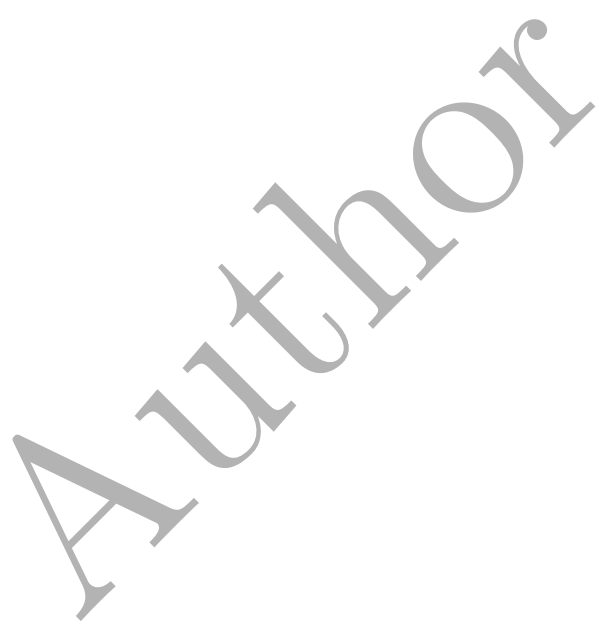

